\title{
Disfunción eréctil: un problema poco valorado en Medicina Interna. Estudio de pacientes y medicación relacionada
}

\author{
I. VILLAMIL CAJOTO, J. A. DÍAZ PEROMINGO, J. SÁNCHEZ LEIRA, F. GARCÍA \\ SUÁREZ, J. SABORIDO FORJAN, M. IGLESIAS GALLEGO
}

Servicio de Medicina Interna. Hospital da Barbanza. Riveira. A Coruña

ERECTILE DYSFUNCTION: A NON SUFFICIENTLY VALUED PROBLEM IN INTERNAL MEDICINE. STUDY OF PATIENTS AND RELATED MEDICATION

\section{RESUMEN}

Introducción: La disfunción eréctil (DE) es habitual y puede estar relacionada con causas psicológicas u orgánicas. La realización de una historia sexual y la exploración física, demuestran una sensibilidad del $95 \%$, y especificidad del $50 \%$ en la determinación de la causa de la DE. Se precisan test diagnósticos adicionales para incrementar la especificidad. No hay información disponible sobre las alteraciones en la función sexual en pacientes de Medicina Interna.

Pacientes y métodos: Entrevistas a pacientes no seleccionados tanto ambulatorios como hospitalizados aplicando el Índice Internacional de Disfunción Eréctil (IIDE) y la Entrevista de Salud Sexual para Hombres (ESSH) durante el año 2003.

Resultados: Se entrevistaron 51 varones de edad media 65 años (3088 ). Entre ellos, 27 negaron actividad sexual (grupo I), la edad media en este grupo fue de 64 años. En los 24 pacientes con actividad sexual (grupo II) la edad media fue de 61 años. El IIDE mostró puntuaciones menores de 45 puntos en 11 pacientes (grupos I y II de disfunción eréctil). La puntuación media para el ESSH fue de 26 puntos. La puntuación fue $<21$ en 10 pacientes $(41,6 \%)$. La concordancia entre ambos test fue del 90,9\%. Los factores de riesgo incluían: tabaco (12 pacientes), alcohol (9), HTA (8) y DM (7).

Conclusión: Parece existir una alta prevalencia de DE entre los pacientes atendidos por Internistas $(41,6 \%)$. Ninguno de éstos recibía tratamiento por esta enfermedad ni habían consultado nunca por ello. La edad media es inferior en los pacientes que mantienen actividad sexual. Consideramos necesario incluir esta patología en la anamnesis a todo paciente asistido para posibilitar la prescripción de tratamiento que podría mejorar notablemente su calidad de vida.

PALABRAS CLAVE: Disfunción eréctil. Varón. Medicina Interna.

\section{ABSTRACT}

Introduction: Erectile dysfunction (ED) is a common disease and may be due to psychological or organic causes. It might point to a silent disease and its value could not only be in life quality but also directly resolving the health problem laying beneath. The evaluation of ED begins with sexual records and physical examination which have been reported to have a 95 percent sensitivity, and a 50 percent specificity. Additional diagnostic tests are needed to maximize specificity. Sexual impairment in general Internal Medicine patients has not been sufficiently studied yet.

Patients and methods: Unselected ambulatory and hospital admitted patients were interviewed during 2003 using the International Index of Erectile Function (IIEF) ad the Sexual Health Interview for Men (SHIM). The cut-off point was 21.

Results: A total of 51 male patients were interviewed, middle age was 65 years old (30-88). Of these, 27 denied sexual activity (group I). Middle age in this group was 64 years old. In the 24 patients with sexual activity (group II) middle age was 61 years old. The IIEF score was less than 45 in 11 patients (groups I and II of erectile dysfunction) The SHIM middle score was 26 points. The score was $<21$ in 10 patients $(41,6 \%)$. Concordance between both test was $90.9 \%$. Risk factors included: tobacco (12 patients), alcohol (9), hypertension (8), and diabetes (7) among others.

Conclusion: Our investigation seems to show that a great number of patients attended by Internists have sexual impairment (41.6\%). None of these were under specific therapy nor consulted for this disease. Middle age is lower among patients with sexual activity as compared with the group without sexual activity. This assessment should be included in the medical interview with the aim of prescribing specific therapy to improve patient's quality of life.

KEY WORDS: Erectile dysfunction. Male. Internal Medicine.

Villamil Cajoto I, Díaz Peromingo JA, Sánchez Leira J, García Suárez F, Saborido Forjan J, Iglesias Gallego M. Disfunción eréctil: un problema poco valorado en Medicina Interna. Estudio de pacientes y medicación relacionada. An Med Interna (Madrid) 2006; 23: 115-118.

\section{INTRODUCCIÓN}

En el pasado, tanto los médicos como la población general consideraban la disfunción eréctil (DE) como una consecuencia inevitable de la edad. Ésta se define como la incapacidad persistente o recurrente para conseguir o mantener la suficien- te rigidez del pene que permita una relación sexual satisfactoria. Se restringe la definición a la capacidad de erección del pene excluyéndose trastornos del deseo sexual, de la eyaculación o del orgasmo (1). Este tradicional error de concepto, unido a la tendencia natural entre los hombres a no reconocer el problema, así como la inexperiencia de los sanitarios en el

Trabajo aceptado: 20 de octubre de 2005

Correspondencia: Iago Villamil Cajoto. Servicio de Medicina Interna. Hospital Clínico Universitario. Choupana, s/n. 15706 Santiago de Compostela, A Coruña. e-mail: iago.villamil.cajoto@sergas.es 
abordaje de los temas sexuales, ha permitido llegar a un cierto clima de nihilismo diagnóstico y terapéutico. Sin embargo, los conocimientos de la función y disfunción sexual masculina son cada vez mayores y hay disponible un arsenal terapéutico importante que es necesario conocer. La prevalencia de esta enfermedad ha sido hasta ahora difícil de precisar, dada la escasa disponibilidad y fiabilidad de datos ya que los hombres suelen ser reticentes a responder a cuestionarios respecto a su función sexual (de hecho en la actualidad se tiende a hablar de disfunción eréctil en sustitución del término impotencia para eliminar todo matiz peyorativo). La OMS ha definido la salud sexual como un derecho humano básico que incluye la capacidad para disfrutar y controlar la conducta sexual, la libertad para que no se inhiba la respuesta sexual ni se perjudique la relación sexual por temor, vergüenza, sentido de la culpa, falsas creencias u otros factores así como la libertad para que las enfermedades orgánicas y otras deficiencias no interfieran con la función sexual ni reproductiva (2). La OMS reconoce la DE como un problema de salud con el mismo grado de discapacidad y severidad que la artritis reumatoide y el angor cardíaco.

Sin ser una patología de riesgo vital, pero al afectar en grado tan importante la calidad de vida y poder ser la primera señal de una enfermedad subyacente grave, consideramos que el conocimiento de esta patología es de interés en los pacientes atendidos en los servicios de Medicina Interna y presentamos los datos de un estudio de corte para evaluar la posible y oculta trascendencia de esta patología.

\section{PACIENTES, MATERIALES Y MÉTODOS}

Se reclutaron de forma consecutiva un total de 51 pacientes varones atendidos tanto en planta de hospitalización como en consultas externas de Medicina Interna. Todos los pacientes fueron entrevistados usando en Índice Internacional de Disfunción Eréctil (IIDE) (3) y la Entrevista de Salud Sexual para Hombres (ESSH) (4). El IIDE se interpretó de la siguiente forma: 0-15 puntos, función eréctil muy deteriorada o ineficaz (grupo I); 16-45 puntos, función eréctil medianamente eficaz (grupo II); 46-75 puntos, función eréctil efectiva (grupo III). Para la ESSH, el punto de corte establecido para el diagnóstico de la disfunción eréctil fue de 21. Se recogieron así mismo los factores de riesgo asociados a deterioro de la función sexual incluyéndose los siguientes: consumo de tabaco, consumo de alcohol, presencia de diabetes mellitus, hipertensión arterial, hipertrofia benigna de próstata, cirugía prostática previa, consumo de psicofármacos (especificándose cuáles), enfermedad cardíaca concomitante, uso de betabloqueantes y conjunción de 2 o más factores de riesgo en un mismo paciente.

\section{RESULTADOS}

La media de edad de los pacientes incluidos en el estudio fue de 65 años con un rango entre 30 y 88 años. De los 51 pacientes entrevistados, 27 negaron mantenerse activos sexualmente y en ellos la media de edad fue de 64 años (30-88 años). En los 24 pacientes con actividad sexual, la edad media fue de 61 años (35-75 años). El IIDE mostró puntuaciones menores de 45 puntos en 11 pacientes encuadrándose en los grupos I y II de interpretación del test y 13 pacientes alcanzaron puntuaciones mayores de 45 puntos perteneciendo al grupo III de función eréctil efectiva. La ESSH obtuvo una puntuación media de 26 (9-53) con 10 pacientes con una puntuación menor o igual a 21 (41,6\%). La concordancia entre ambos test fue pues del 90,9\%. Sólo se evidenció discordancia en 1 paciente con respecto a ambos test. Ningún paciente estaba recibiendo tratamiento específico para la disfunción eréctil. Con respecto a los factores de riesgo asociados para el grupo de pacientes sin actividad sexual, estos fueron: tabaco (5 pacientes), alcohol (3), diabetes (3), HTA (4), hipertrofia benigna prostática (4), cirugía prostática (1), psicofármacos (2, uno sertralina y uno mirtazapina), enfermedad cardíaca (3) y uso de beta-bloqueantes (1). Siete pacientes presentaban 2 o más factores de riesgo asociados (Fig. 1). En el grupo de pacientes con mantenimiento de la actividad eréctil de forma parcial o completa, los factores de riesgo asociados fueron: tabaco (7 pacientes),alcohol (6), diabetes (4), HTA (6), hipertrofia benigna de próstata (5), cirugía prostática (3), psicofármacos (1, fluoxetina), enfermedad cardíaca (4) y uso de beta-bloqueantes (2). En 12 pacientes se asociaban $2 \mathrm{o}$ más factores de riesgo (Fig. 2).

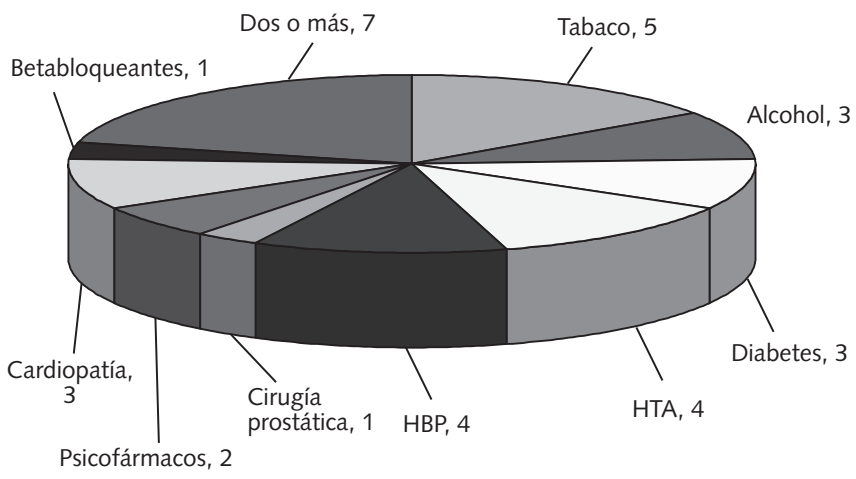

Fig. 1. Factores de riesgo asociados en los pacientes sin actividad sexual.

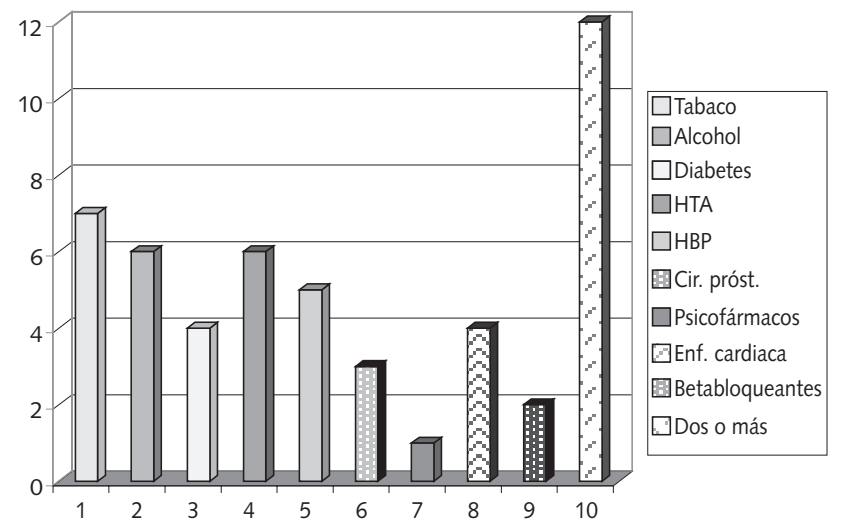

Fig. 2. Factores de riesgo en el grupo de pacientes con actividad sexual.

\section{DISCUSIÓN}

La DE es en definitiva un problema de salud. A diferencia del concepto tradicional casi teleológico que hacía de esta circunstancia una mera "consecuencia de la edad", hoy es consi- 
derada como una patología. Se trata además de una enfermedad bastante prevalente, tras haberse superado el sesgo que hacía que los individuos consultados habitualmente no fueran sinceros. En el informe Kinsey de 1948, por ejemplo, parecía que la impotencia solo afectaba al $10 \%$ de los varones adultos con una mayor prevalencia en las edades mayores de 75 años (5). En comparación, la información de un estudio de 1994, el Massachusetts Male Aging Study (MMAS), realizado entre 1290 varones de edades comprendidas entre los 40 y los 70 años, indica una prevalencia de DE de cualquier grado del $52 \%$ y revela que la disfunción sexual masculina presente como DE, disminución de la líbido o eyaculación anormal aparece por primera vez a partir de los 40 años y empeora con la edad avanzada. A los 40 por ejemplo, el $40 \%$ reconoce algún grado de empeoramiento de la función sexual y otro $10 \%$ reconoce disminución de la capacidad o deseo sexual con cada década (6). En España el estudio EDEM (Epidemiología de la Disfunción Eréctil Masculina) (7), realizado entre 2476 varones de entre 25 y 70 años de edad, encontró algún grado de DE en el $12 \%$, de lo que se deduce, ajustándolo al censo de población masculina, una prevalencia estimada de DE en España de 1.500.000 a 2.000.000 de varones. Si esto es así, el $17,7 \%$ de los varones españoles padecen DE, lo que indicaría una prevalencia en España inferior a la detectada en EE.UU. Las causas de la DE se resumen en la Tabla I.

Los datos de nuestro estudio indican un alto porcentaje de inactividad sexual (27 pacientes, 53\%) de entre los que podemos asumir que en algunos casos al menos podría estar relacionado con DE (aunque no se obtuvo más información en

\section{TABLA I}

PRINCIPALES CAUSAS DE DISFUNCIÓN ERÉCTIL

Causas orgánicas
Causas vasculares (60-80\%)
Arterioesclerosis: hiperlipemia, HTA, DM
Enfermedad de la Peyronie
Fracturas pélvicas, traumatismo perineal
Fractura de cuerpos cavernosos
Trasplante renal
S. de Leriche, by-pass aorto-ilíaco o aortofemoral
Secuelas de Radioterapia o de priapismo
Causas neurológicas (10-20\%)
Sistema nervioso central: ECVA, SAOS, enfermedad de Alzheimer,
enfermedad de Parkinson, tumor cerebral
Médula espinal: Traumatismos, compresión, tumores, enfermedad
desmielinizante.
Sistema nervioso periférico: neuropatía (diabética, alcohólica),
secuelas postquirúrgicas
Causas hormonales (5-10\%)
Exceso de estrógenos: exógenos, hepatopatía, tumor secretor de
estradiol o HCG
Hiperprolactinemia: iatrogénica, tumor hipofisario
Hipogonadismos: hipogonadotróficos, hipergonadotróficos
Disfunción tiroidea: hipertiroidismo, hipotiroidismo.
Disfunción suprarrenal: síndrome y enfermedad de Cushing, insufi-
ciencia suprarrenal
Malnutrición severa.
Causas farmacológicas (Tabla II)
Consumo de tóxicos/drogas (tabaco, alcohol, drogas ilegales, etc.)
Trastornos afectivos

TABLA II

FÁRMACOS ASOCIADOS A DISFUNCIÓN ERÉCTIL (9)

Familia farmacológica

Antihipertensivos

Diuréticos: espironolactona, tiazídicos, antialdosterónicos

Simpaticolíticos: guanadrel, clonidina, metildopa, reserpina, guanetidina, clortalidona

Bloqueantes beta adrenérgicos: propranolol, betapindol, atenolol, metoprolol, labetalol

Hidralazina

Metirosina, prazosin

Fármacos psicolépticos, psicoanalépticos

Antipsicóticos: fenotiazinas, barbitúricos, haloperidol, tioridazina

Ansiolíticos: $\quad$ alprazolam, diazepam, lorazepam, clozapina

Antidepresivos: amitriptilina, carbamacepina, litio, maprotilina, fenelcina, trazodona

Citotóxicos

Metotrexate, nafarelina, leuprorelina, tamoxifeno

Fármacos de acción hormonal

Antiandrógenos, estrógenos, esteroides, anabolizantes (danazol), ketoconazol, digoxina, clofibrato, cimetidina

Otros

Anticolinérgicos, disulfiram, ranitidina, famotidina, omeprazol, gemfibrocilo, indometacina, interferón alfa, levodopa, metildopa, fenitoina, fenobarbital, pergolida, naproxeno, sulfasalazina, etc.

este sentido). Entre los pacientes activos sexualmente el 45,8\% (11 pacientes) presentan índices de disfunción sexual ineficaz o sólo medianamente eficaz. Por tanto, la prevalencia de la disfunción sexual puede ser más elevada de la estimada. En nuestro estudio, los dos test utilizados mostraron una elevada concordancia por lo que, dado que la ESSH es más rápida en su realización, podría recomendarse realizar el screening con la ESSH y en casos con puntuaciones cercanas el punto de corte (21 puntos) utilizar el IIDE.

Además de información oculta que podría haber estado infraestimando la prevalencia, existen factores de riesgo que probablemente jueguen un papel importante en un posible incremento de la incidencia actual de DE. Así, además de los factores psicógenos a los que se dio mas interés en los años 50 a 70 del siglo pasado, son de especial interés en la población valorada por Medicina Interna las causas orgánicas, asumiéndose en la conferencia de consenso del NIH de 1993 que probablemente estas serían responsables de la mayor parte de las DE. Por otro lado, la DE puede permitir un diagnóstico precoz de una enfermedad subyacente, desde la hipertrofia prostática, benigna o no, hasta la enfermedad cardiovascular (la incidencia de DE tras IAM se sitúa entre un 38 y un 78\%) (8), pasando por la diabetes mellitus. Así, algunos estudios estiman una prevalencia del $50 \%$ de DE en estos pacientes diabéticos debido a la existencia de microangiopatía (9). Nuestros pacientes presentan un porcentaje importante de factores de riesgo de DE por lo que el papel de la DE en el diagnóstico precoz de la patología responsable de ésta parece trascendente. 
Este estudio presenta limitaciones. En primer lugar, no pueden hacerse inferencias en la población general por la escasa muestra del estudio, pero es una aproximación a este grupo de pacientes en nuestra especialidad. En segundo lugar al tratarse de un estudio transversal, no se aportan datos de seguimiento a largo plazo, ni de estudio de las causas en profundidad, pero el objetivo del estudio pretendía evidenciar un fotograma de la situación exclusivamente. Por último la mencionada cifra de pacientes entrevistados sin actividad sexual en los que no se pudo determinar su relación con DE. Tenemos especial interés en destacar el consumo de fármacos relacionados con DE (Tabla II) (10), muchos de los cuales ni siquiera recogen este efecto secundario en ficha técnica y muchos otros que tampoco se consideran. Por ejemplo, asumiendo que los beta-bloqueantes provocan $\mathrm{DE}$, pocas veces se valora el efecto de los beta adrenérgicos (o se cree que no provocan alteraciones eréctiles) pero resulta que terbutalina y salbutamol (de alto consumo en general en Medicina Interna) son tratamiento de indicación en el priapismo (11) con el consiguiente efecto potencial en la DE. Llama la atención en nuestros resultados el alto grado de "resignación" de los pacientes -y sus parejas- que sólo verbalizan este problema si es puesto sobre la mesa, por lo que debería estar presente en las anamnesis habituales.

En definitiva, nuestros datos ponen de manifiesto una muy importante prevalencia de la enfermedad, un alto grado de subestima por parte de los pacientes y de los médicos (ningún paciente había consultado el problema y, siendo la mayoría pacientes con patología crónica, ningún médico lo había estimado) con una importante trascendencia en calidad de vida y un potencial valor indicativo de patología subyacente silente no contemplada, por lo que consideramos necesario valorar esta enfermedad con un test sencillo, de aplicación rápida, que puede contribuir a mejorar la calidad de vida y detectar precozmente enfermedades subyacentes o efectos secundarios de medicación, en algún caso evitables.

\section{Bibliografía}

1. NIH Consensus Conference. Impotence. NIH Consensus Panel on Impotence JAMA 1993; 270: 83-90.

2. Gro Harlem Brundtland. The Word health report 1999. Disponible en línea http//:ftp.who.int/gb/pdf_files/WHA52/ew4.pdf. (última consulta 31 mayo2005).

3. Rosen RC, Riley A, Wagner G, Osterloh IH, Kirpatrick J, Mishra A. The International Index of Erectile Dysfunction (IIED): a multidimensional scale for assessment of erectile dysfunction. Urology 1997; 49: 822-830.

4. Cappelleri JC, Siegel RL, Glasser DB, Osterloh IH, Rosen RC. Relationship between patient self-assessment of erectile dysfunction and the sexual health inventory for men. Clin Ther 2001; 10: 1707-1719.

5. Kinsey, AC. Sexual behaviour in the human male. Saunders, Philadelphia, 1948.

6. Feldman, HA, Goldstein, I, Hatzichristou, DJ, et al. Impotence and its

medical and psychosocial correlates: Results of the Massachusetts male ageing study. J Urol 1994; 151: 54.

7. Martín Morales A, Sánchez Cruz JJ, Sáenz de Tejada I, Rodríguez Vela L, Jiménez Cruz JF, Burgos-Rodríguez R. Prevalence and independent risk factors for erectile dysfunction in Spain: results of the Epidemiología de la Disfunción Eréctil Masculina Study. J Urol 2001; 166: 53-58.

8. De Busck R, Dorry Y, Goldstein I, jackson G, Kaul S, Kimmel SE et al. Management of sexual dysfunction in patients with cardiovascular disease: recommendations of the Princeton Consensus Panel. Am J Cardiol 2000; 86: 175-181.

9. Drugs that cause sexual dysfunction: an update. The Medical Letter 1992; 34:73-8

10. Morgentaler A. Male Impotence. Lancet 1999; 354: 1713-1718.

11. Vilke GM, Harrigan RA, Ufberg JW, Chan TC. Emergency evaluation and treatment of priapism. J Emerg Med 2004; 26: 325-9. 